Louisiana State University

LSU Digital Commons

Faculty Publications

Department of Biological Sciences

7-30-2004

\title{
Visualizing chromosome structure/organization
}

Eric Lam

Rutgers University-New Brunswick

Naohiro Kato

Rutgers University-New Brunswick

Koichi Watanabe

Rutgers University-New Brunswick

Follow this and additional works at: https://digitalcommons.Isu.edu/biosci_pubs

\section{Recommended Citation}

Lam, E., Kato, N., \& Watanabe, K. (2004). Visualizing chromosome structure/organization. Annual Review of Plant Biology, 55, 537-554. https://doi.org/10.1146/annurev.arplant.55.031903.141807

This Article is brought to you for free and open access by the Department of Biological Sciences at LSU Digital Commons. It has been accepted for inclusion in Faculty Publications by an authorized administrator of LSU Digital Commons. For more information, please contact ir@lsu.edu. 


\title{
Visualizing Chromosome StruCture/Organization
}

\author{
Eric Lam, Naohiro Kato, and Koichi Watanabe \\ Biotech Center for Agriculture and the Environment, Department of Plant Science, \\ Rutgers the State University of New Jersey, New Brunswick, New Jersey 08901; \\ email:ericl89@hotmail.com,kato@aesop.rutgers.edu,kowatana@aesop.rutgers.edu
}

Key Words chromatin, interphase, FISH, GISH, green fluorescent protein tagging

- Abstract With the rapid development of sequencing technologies in the past decade, many eukaryotic genomes have been resolved at the primary sequence level. However, organization of the genome within nuclei and the principles that govern such properties remain largely unclear. Optimization of fluorescence probe-based hybridization technologies combined with new advances in the instrumentation for microscopy has steadily yielded more structural information on chromosome organization in eukaryote model systems. These studies provide static snapshots of the detailed organization of chromatin. More recently, the successful application of a chromatin tagging strategy utilizing auto fluorescent fusion proteins opened a new era of chromatin studies in which the dynamic organization of the genome can be tracked in near real time. This review focuses on these new approaches to studying chromatin organization and dynamics in plants, and on future prospects in unraveling the basic principle of chromosome organization.

\section{CONTENTS}

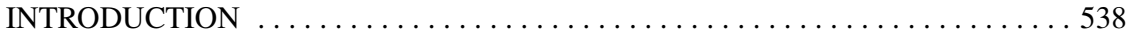

CHROMATIN AS THE BASIC COMPONENT OF CHROMOSOMES $\ldots \ldots \ldots 539$

Composition of Chromatin and its Macromolecular Structure ............. 539

Chromatin Structure and Genomic Information Processes ................ 540

TECHNOLOGIES FOR VISUALIZING DNA AND CHROMATIN .......... 542

Classical Cytological Approaches and DAPI Staining

for Heterochromatin ................................ 542

FISH: Sequence-Specific Detection of DNA Via Hybridization $\ldots \ldots \ldots \ldots . \ldots 543$

Fluorescent dUTP Incorporation: Chromosome Territories

in Living Cells . ..................................... 545

Fluorescent Protein-Based Chromatin Tagging . . . . . . . . . . . . . . 545

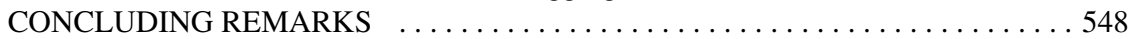




\section{INTRODUCTION}

The packing of chromatin within the eukaryote nucleus has been a subject of great interest since the discovery that DNA contains the information that controls the makeup and function of the cell. Together with the genomic DNA of the cell and heterogeneous nuclear RNAs (hnRNAs), there are a large variety and abundance of proteins in the nucleus. The high concentration of macromolecules and proteins within the nucleus is manifested in its high density, and diffusion of chromatin within the nucleoplasm is typically limited. These characteristics raise the speculation that for processes such as transcription and recombination to operate efficiently, nuclear DNA must be organized in a structured fashion (56, 64). A structured nucleoplasm is supported by observations that suggest an unequal distribution of certain transcription factors and splicing factors within the nucleus of interphase cells (69) and the tracking of transcript movement from the nucleus to the cytoplasm (56). However, direct demonstration and characterization of chromatin organization in the nucleus are extremely difficult. Early observations by Rabl (29) suggested that plants have a preferentially polarized organization of centromeres clustered at one end of the nuclear envelope (the apical side) and telomeres at the opposite end (the basal side). The organization of the rest of the chromatin is harder to define. For the past 20 years, optimization of the Fluorescence in situ Hybridization (FISH) techniques has helped to provide a lowresolution picture for disposition of specific DNA sequences within nuclei (86). Combining 3-Dimensional (3-D) fluorescence microscopy and computer-aided deconvolution techniques, some principles of chromatin organization in interphase cells have emerged from studies with mammalian and Drosophila cells: (a) Each individual chromosome occupies a discrete space (a so-called "territory") in the nucleus with little intertwining among the other chromosomes $(41,42,81,94)$. (b) Interactions with the nuclear envelope and between heterochromatic regions are important factors that contribute to the organization of the subnuclear environment (64). Consistent contact points between chromosomes and the nuclear envelope were observed in Drosophila, thus arguing for nonrandom distribution of chromatin within the nuclei (62). (c) Chromosome movements during meiosis and mitosis are directed and may be nonrandom $(17,29,40,70)$. A quantitative understanding of how chromatin is organized at various scales of resolution is ultimately necessary to unravel the complex roles that chromatin states can impose on control of nuclear processes, such as transcription and DNA replication, both locally and globally. With the advent of genome sequencing and DNA labeling technologies, it is now possible to integrate genetic and molecular data with physical information related to chromatin organization at an unprecedented level of resolution. This review summarizes our current state of progress in deploying these novel techniques in model plant systems and hopes to stimulate as well as to contribute to more future refinement and advances in the quantitative description of chromatin organization and its relevance to nuclear processes. 


\section{CHROMATIN AS THE BASIC COMPONENT OF CHROMOSOMES}

\section{Composition of Chromatin and its Macromolecular} Structure

Chromosomes are the discrete units of the genome that carry all the DNA and genes encoded by it, as well as all the associated proteins. Morphologically they are visible only during particular phases of the cell division cycle in which they are highly condensed. During the interphase of the cell cycle, chromosomes cannot be observed and the mass of DNA protein is collectively known as chromatin. The fundamental unit of chromatin consists of nucleosomes (reviewed in 25, 52), which are formed from a protein core of basic proteins called histones with $\sim 165$ base pairs (bps) of superhelical DNA wound around twice. Each nucleosome is connected to its neighbors by short DNA segments of 10-80 bps called "linker DNA." Variability of the linker length may be related to the regulatory functions that control the particular locus. Each histone core is composed of eight polypeptides, two each of four highly conserved proteins: histones H2A, H2B, H3, and H4. Together, this "octamer" of histone proteins provides the first level of organized compaction for nuclear DNA. In addition to this structural role, it also creates a barrier for accessibility for nuclear enzymes such as transcription factors to locate their target site of action. The linear arrangement of nucleosomes with their linker DNA forms the classic "beads on a string" model of nuclear DNA organization and has a compaction ratio of about 5- to 10-fold (i.e., from 2-nm diameter of "naked" superhelical DNA to about $11 \mathrm{~nm}$ ). The second level of organization is the folding of nucleosomes into 30-nm chromatin "fibers," which further compact the DNA by another 10-fold. A fifth histone, histone H1, stabilizes this 30-nm fiber form of chromatin by binding to the linker DNA and to the nucleosomes. Beyond this level of chromatin organization, the picture is less clear. Based on more recent work, it is likely that chromatin can be further compacted in highly condensed regions of the genome to larger structures with diameters of 300-700 $\mathrm{nm}$ (25). These condensed regions are typically rich in repetitive sequences and are much less accessible to nuclear enzymes for replication or transcription. In contrast, active regions of the genome rich in single-copy genes will likely form loops of up to several megabase pairs (Mbps). A key characteristic of the nucleosome unit is its inherent dynamic nature. The linker histone $\mathrm{H} 1$ has an on/off rate of seconds, and the core histones are much more stable with exchange rates measured at orders of minutes (for $\mathrm{H} 2 \mathrm{~A} / \mathrm{H} 2 \mathrm{~B}$ ) to hours (for $\mathrm{H} 3 / \mathrm{H} 4)$. Covalent modification of the histones by various enzymes, especially at their amino-terminal "tails" that extend out of the histone octamer core, can significantly alter the stability of the nucleosome unit and is important to understanding how nuclear processes such as transcription can be controlled locally and globally. 


\section{Chromatin Structure and Genomic Information Processes}

The compartmentalization of DNA within the nucleus of eukaryotic cells requires a compaction of DNA. Transformation of DNA fiber to chromatin fiber and chromosomes fulfills this requirement, as discussed above. The chromosomes of eukaryotes are characterized by the presence of deeply staining material, heterochromatin, which is distinguishable cytologically from the surrounding lightly staining euchromatic regions $(16,43)$. From studies of position effects on transcription activity, heterochromatin is transcriptionally inactive and euchromatin contains most of the active genes in a genome. This view of heterochromatin is changing due to the finding that the repeats within centromeres are transcribed, and these transcripts produce siRNAs (90). Many repetitive sequences are identified in heterochromatin regions. In Arabidopsis, heterochromatin is largely confined to the pericentromeric regions in each of the five chromosomes and to the nucleolus organizer regions (NORs). NORs include thousands of kilobases of tandemly repeated ribosomal RNA-encoding genes $(18,19)$, and pericentromeric regions include 180-bp satellite repeats, as well as retrotransposons, most notably from the Athila family (34, 72, 76). Cytosine methylation and distinctive histone modifications are frequent on repeat sequences, and these kinds of chromatin modifications are involved in heterochromatin organization.

During DNA replication, epigenetic information is transferred onto the daughter DNA strand thorough DNA methylation and histone modifications. Dnmt1-type DNA methylase adds methyl-residues to cytosines on newly synthesized strands by using the information on the parental DNA strands, whereas Dnmt3-type DNA methylase functions in de novo DNA methylation. Plants have both types of DNA methyltransferase. Chromomethylase is the only DNA methylase currently known to be unique to plants, and chromomethylases in Arabidopsis and maize recognize CpNpG sequences $(57,71)$. Histones undergo covalent modifications including acetylation, methylation, phosphorylation, and ubiquitination on mainly their $\mathrm{N}$ terminal tails $(25,58)$. These modifications can result in structural change of chromatin fibers, and the interrelationship among each histone modification is now clear. Histone methylation shows a close relationship with heterochromatin organization. Several kinds of enzymes are currently shown to carry out histone methylation on distinct sites, and methylation at each position implies a distinct biological consequence. Histone H3 Lys4 (K4) methylation correlates with gene activation, whereas histone H3 Lys9 (K9) methylation is preferentially correlated with gene repression and is associated with heterochromatin. Heterochromatin protein 1 (HP1) binds with high affinity to Lys9-methylated histone $\mathrm{H} 3$, and oligomerization of HP1 plays a role in heterochromatin formation $(9,55)$. In mammal and fission yeast, $\mathrm{Su}(\mathrm{var}) 3-9$-type histone methyltransferases interact with HP1 homologs $(24,78)$. These in turn lead to the recruitment of a histone methyltransferase and expansion of the $\mathrm{H} 3 \mathrm{~K} 9$ modification to neighboring nucleosomes and inactivation of gene activity in the locus.

It is unclear how epigenetic information on histones is transferred from the parental to daughter chromosomes. DECREASED DNA METHYLATION 1 
(DDM1) could be involved in maintaining information on nucleosomes in Arabidopsis. The mutant of DDM1, which encodes an ATP-dependent chromatin remodeling factor (46), shows reduced levels of genomic DNA methylation, particularly on repetitive sequences, and Lys9-methylated histone $\mathrm{H} 3(30,47,91)$. DAPI (4', 6-diamidino-2-phenylindole) staining analysis (discussed below) and fluorescence immunostaining analysis using antibodies against methylated histone $\mathrm{H} 3$ show that the $d d m 1$ mutation results in decreased clearly stained foci by DAPI and an antibody against Lys9-methylated histone H3, indicating decondensation of centromeric heterochromatin $(74,82)$. The mutant of DNA methtyltransferase 1 (met 1 ) also shows a reduction of heterochromatic chromocenter (82, 85). DDM1 and MET1 could control heterochromatin assembly at chromocenters by regulating DNA methylation and following histone H3 K9 methylation.

The two processes, histone modification and DNA modification, could be intimately linked in some cases via protein-protein interaction $(25,30)$. Localization of Dnmt3a with HP1 has been observed in mammalian cells (7), whereas CHROMOMETHYLASE 3 (Cmt3) DNA methylase interacts with the heterochromatin protein of Arabidopsis, LIKE HETEROCHROMATIN PROTEIN 1 (Lhp1). Histone H3 K9 methylation by KRYPTONITE, Arabidopsis histone H3 K9 methylase, results in recruitment of Cmt3 methylase through the interaction of Lys9methylated histone $\mathrm{H} 3$ with Lhp1, and it is different from the direct interaction of HP1 with histone H3 K9 methylase seen in S. pombe and animals (45). Recent evidence from S. pombe and Arabidopsis suggests that the processes of RNA interference/cosuppression, DNA methylation, and heterochromatin-directed gene silencing may all be interrelated through modifications in chromatin structure (6, $33,90)$.

Chromatin organization is clearly maintained by multiple types of interactions between chromatin fibers and chromatin modifiers (i.e., epigenetic memory). It is presently unclear whether nucleosome modifications are involved in the global organization of chromosomes within the nucleus, and how the particular subnuclear location may in turn affect the stability of histone/DNA interaction. Some initial points of reference are likely established early in the life of a cell after it has completed mitosis. The Rabl configuration likely sets up the initial state of chromatin organization. However, after this initial state, organization of the interphase nucleus is much less defined. It is now clear from studies of various systems that each chromosome occupies a discrete "territory" in the nucleus, and radial positioning of a chromosome in the nucleus from interior to periphery has an intimate correlation with the size and gene density of each chromosome $(21,65)$. This suggests mechanism(s) that act to define the position of each chromosome within the nucleus. One candidate for mediating nuclear organization could be specialized regions of genomic DNA called matrix attachment regions (MARs) and proteins associated with MARs, which help establish stable sites of contact between chromatin and the nuclear envelope to mediate genome organization at a global level. The components of the nuclear envelope might be involved in nuclear organization and dynamics of chromatin and chromosomes with the regulator 
proteins like the small GTPase Ran in plants $(3,68,77)$ as animal counterparts $(5,8$, 38). Currently, to what extent this order is maintained from nucleus-to-nucleus and whether there is a set of predictable routes through which the genome can reorganize itself after the initial Rabl state is unclear. The ability to track specific loci of the genome in the nuclei of interphase cells is necessary to address these fundamental questions.

\section{TECHNOLOGIES FOR VISUALIZING DNA AND CHROMATIN}

\section{Classical Cytological Approaches and DAPI Staining for Heterochromatin}

In plants, the technique of chromosome spreading dominated most of the earlier work on subnuclear order until the advent of electron microscopy approaches (40). The most classical approach to visualizing chromatin is through DNA-binding dyes such as Giemsa stain, aceto-carmin, and aceto-orcein. These dyes have relatively low sensitivity for smaller chromosomes and they do not provide much information about the particular sequences, although their reproducible staining patterns for particular chromosomes enable karyotyping and characterization of relatively large changes in genome organization such as rearrangements and breakage of chromosome arms.

DAPI is a water-soluble fluorescent dye that shows an absorption maximum at $359 \mathrm{~nm}$ (UV region) and an emission maximum at $461 \mathrm{~nm}$ (blue color). It is semipermeable to live cells and thus can be used with both live and fixed cells. DAPI associates with the minor groove of double-stranded DNA preferentially to AT-rich regions, and its fluorescence intensity increases about 20 times after binding to DNA due to displaced water molecules from both DAPI and DNA (10, 53, 67). DAPI unevenly stains chromosomes because AT-rich sequences are differentially distributed over their lengths. Repetitive DNA such as pericentromeric heterochromatin and nucleolar organizer regions (NORs) is usually highly condensed and contain predominantly AT-rich sequences. Therefore, DAPI fluorescence intensities in such regions are much higher than in other euchromatic regions. In Arabidopsis, which has only about $10-12 \%$ repetitive sequences in the genome, the highly stained regions appear as speckles in interphase nuclei, and these correspond to "chromocenters" where heterochromatin exists in a highly condensed state near the centromeres. Assigning these chromocenters is possible by using hybridization techniques with bacterial artificial chromosome (BAC) clones that are mapped to regions of the genome adjacent to the NORs or neighboring the centromeres [(27); see below for discussion on FISH techniques]. DAPI staining is frequently used to identify basic chromosome organization in many organisms including plants. 


\section{FISH: Sequence-Specific Detection of DNA Via Hybridization}

FISH is an in situ DNA detection technique in which fluorescent dyes are conjugated to DNA probes (73). To detect a specific DNA sequence in chromosomes in nuclei, the samples are usually fixed and denatured before hybridization to the DNA probe that contains the specific sequence. To make the DNA probe permeable to the nucleoplasm, one must at least partially remove the cell wall and cytosol from plant samples of interest by either mechanical (including a flow sorting machine) or chemical (such as enzymatic digestions) methods before denaturing the DNA in the samples. The choice of fluorescent dyes is numerous, with many spectrally distinct chromophores spanning the optical range of UV to near infrared available commercially. Fluorescence microscopes that can precisely distinguish many of these dyes have also been developed. By combining these technical advances, one can detect many different DNA sequences in a single nucleus. To date, the most colors of fluorescent dye combinations in a single cell that have been achieved are 27 for human chromosomes $(79,83)$, but more dye combinations are theoretically possible by combining more color variants and DNA probes.

Most intensive work employing the FISH technique to study chromosome organization in plants has occurred in maize (Zea mays) and other grasses using telomere and centromere probes containing repetitive sequences (11; reviewed in $22,36,37)$. Work from the Cande group shows that telomeres are randomly scattered in the maize nucleoplasm excluding the nucleolus in interphase nuclei (11, 15). Once cells enter meiosis, telomeres are relocated to the nuclear periphery and apparently slide on the nuclear envelope during late Leptotene and Zygotene stages to make a telomere cluster, the so-called "bouquet." This movement plays a crucial role in controlling chromosome pairing or crossover. In wheat, the Phl locus is genetically proved to be important for correct pairing and recombination. FISH reveals that $P h l$ locus reduces nonhomologue association at their centromeres (66). Recently, the Cande group adapted a computational model to the FISH analysis data obtained from rye anthers showing that the observed telomere movement is a directed active process (15). In Arabidopsis, Fransz and coworkers combined chromosome-specific FISH using BACs neighboring pericentromeric regions or NORs and DAPI staining of chromocenters and showed that these condensed repetitive regions can interact at a high frequency in interphase cells (27). Transcription of rDNA units and the associated regions of the NOR have also been characterized at high resolution using a combination of FISH and electron microscopy (EM) techniques. By incorporating Bromo-UTP into thin root sections of pea, active transcription sites within the nucleus can be visualized. Together with FISH probes that are amenable to subsequent immunogold detection, genomic sequences corresponding to the rDNA repeats can be simultaneously visualized in EM sections $(2,32)$.

By extending the FISH technique entire chromosome(s) can be specifically decorated, a technique called "chromosome painting" $(79,95)$. In this procedure 
each chromosome is isolated by flow sorting or microdisection from tissues or cells of interest. The isolated chromosomes are then used as templates for polymerase chain reaction (PCR) with degenerated primers and fluorescent derivatives of dCTP ( $2^{\prime}$-deoxycytosine $5^{\prime}$-triphosphate) to produce DNA probes (80). A single fluorescent derivative of dCTP or a mixture of dCTP derivatives with distinct fluorescent labels can be used to create chromosome-specific probes of different colors. The probes are hybridized to target samples such as permeablized, fixed nuclei or chromosome spreads. Typically, unlabeled DNA fragments containing disperse repeat sequences from the target chromosomes are included to increase specificities of the probes by suppressing cross-chromosome hybridization due to the presence and abundance of these repetitive sequences all over the genome. Chromosome painting is widely used in mammals, birds, and insects to characterize chromosome arrangements. This technique revealed that each chromosome in interphase nuclei is not entangled with the others but occupies a discrete territory (see above and also reviewed in 20, 21, 95). Because of the abundance of dispersed repetitive DNA sequences throughout the genome in most plant species, chromosome painting in plants has been limited to unusual chromosomes such as the so-called SUPERNUMERARY or B chromosomes, which exist only in limited species (44). Chromosomes in several hybrid plants that carry chromosomes from different species, such as the hybrid of barley and rye, can be painted. To paint alien chromosomes, one or both parental chromosomes are extracted and used as probes. This technique is called genomic in situ hybridization (GISH), and mainly has been used to characterize the hybrid status of progenies from a cross of two distinct parental species (23). In some cases, there is clear segregation of the two genomes into distinct and separate domains within the nuclei of the hybrid. In hybrids where a strong concentric arrangement of the two genomes was observed, the phenotype of the hybrid progeny resembles the parent that contributed to the outer whorl genome $(12,35)$. This latter result is especially intriguing because it provides evidence that nuclear location may determine gene activity. A similar differential subnuclear localization of the nucleoli of the two genomes may account for the Nucleolar Dominance phenomenon (40).

Recently, Fransz and others established chromosome painting in Arabidopsis $(27,59,60)$, taking advantage of the tremendous molecular, genetic, and genomic resources that have been accumulated within the last few years. Using publicly available BAC clones that span the length of the chromosome of interest, and carefully eliminating clones that contain dispersed repeated sequences, chromosome-specific FISH probes were constructed and used to characterize their behavior in interphase or meiosis. Their results suggest that Arabidopsis chromosomes also occupy territories in interphase nuclei, and euchromatic regions of a chromosome emerge from a chromocenter and create loops while highly condensed heterochromatin often associates at chromocenters. In contrast to observations in maize, telomeres of Arabidopsis are not scattered in the nucleoplasm but are clustered around the nucleolus. I. Schubert's group is currently expanding this technique (Figure 1) to paint multiple chromosomes of 
Arabidopsis simultaneously, as was achieved in human cells (I. Schubert, personal communication).

\section{Fluorescent dUTP Incorporation: Chromosome Territories in Living Cells}

Formation of chromosome territories can be observed in living mammalian cell cultures by pulse labeling of newly synthesized DNA $(14,92)$ with fluorescentlabeled dUTP ( 2 -deoxyuridine 5 '-triphosphase) delivered into cells by microinjection or by scratching the cells with a needle. The cells are then left to divide several times in a normal culture medium. The chromosomes incorporate the labeled dUTP during DNA replication as the cells undergo mitosis (S-phase) in the first cell cycle, and sister chromatids are labeled. Subsequent cycles of random segregation of labeled and unlabeled chromatids into daughter cells result in a few labeled chromosomes in most nuclei. This technique of pulse labeling with dUTP derivatives shows little effect on the morphology, growth rate, and viability of the cells. For Cy3-dUTP incorporation, the cells show strong fluorescence several days after labeling. An advantage of this approach is that one can directly quantify chromosome motions in a single cell. By using a microscope capable of recording three-dimensional movement as a function of time of single particles, different levels of diffusion motions of chromosome territories were revealed (14). Because free dye-labeled nucleotides are rapidly depleted from the cells, most likely through their rapid incorporation to cytoplasmic vesicles, only parts of chromosomes that are replicated during the injection of nucleotides are labeled. Therefore, one can specifically label certain regions of the genome (i.e., early- and late-replication regions). By optically observing labeled single cells, this approach can track the process of chromosome territory formation and the results show that the territories are composed of subcompartments with diameters of about $500 \mathrm{~nm}$. Early-replicated DNA and the DNA that result from later duplication events form separate compartments. The drawback of this technique is that a whole chromosome is not painted, and usually only one of the two sister chromatids is labeled. The sequence of the painted regions is also difficult to identify. To date, studies utilizing this method with plants have not been reported. However, this could be a feasible approach because microinjection in plants is well established.

\section{Fluorescent Protein-Based Chromatin Tagging}

Although the FISH technique has added valuable insights to our appreciation of subnuclear order, it is an invasive technique that cannot be applied to live cells. Thus, chromatin needs to be denatured and hybridized with labeled probes in order to visualize the position of specific sequences. The concern with artifacts can be easily appreciated. Recently, advances in the application of the Green Fluorescent Protein (GFP) as an in vivo tag have opened many exciting novel avenues of investigation. Using GFP fused to Histone H2B, Kanda et al. (48) first demonstrated in mammalian cells that stable labeling of chromatin and chromosomes can 
be achieved in live cells. In plants, Boisnard-Lorig et al. (13) demonstrated that expression of an H2B fusion protein with Yellow Fluorescent Protein (YFP) in transgenic Arabidopsis plants does not cause obvious developmental defects and this fusion could be used as a noninvasive label of chromatin. They also showed that the fluorescence behavior of this fusion protein in the nucleus during nuclear division correlates with the predicted pattern of dramatic condensation during metaphase. Using these characteristics, one can then track the mitotic activity distribution in the developing endosperm using time-lapse Confocal Microscopy. Gerlich et al. (31) and Walter et al. (92) reported a recent further development of the H2B-fluorescent protein fusion approach. Taking advantage of the slow exchange rate of $\mathrm{H} 2 \mathrm{~B}$ from the nucleosome core, they used a photobleaching approach with $\mathrm{H} 2 \mathrm{~B}-\mathrm{YFP}$ or H2B-GFP expressing mammlian cell nuclei to mark and track specific regions of the genome. Their results indicate that global positions of chromosomes may be heritable through the cell cycle in some cells, and that large-scale movements of chromatin at the scale of several microns can be observed in early G1. Deploying this strategy with H2B::YFP-expressing transgenic plants should be an interesting avenue of investigation in the near future.

In addition to labeling chromatin through fluorescent protein tagging of the nucleosome components, a novel strategy with GFP has also been developed in recent years to track specific chromosomal regions, and it promises to revolutionize our capability to observe chromatin-based processes in real time. The technique (illustrated in Figure 2), originally studied in the laboratory of Andrew Belmont and collaborators, entails the construction of a fusion protein between GFP and the DNA-binding domain (DBD) of a known transcription factor. Next, the binding site for this particular DBD is multimerized to about 250 copies and is then inserted into the genome of animal and yeast cells $(75,84)$. Expression of the GFP-DBD fusion results in fluorescent tagging of the concatamer in situ. This essentially provides a "beacon" that allows one to track the position of this region in the genome specifically. In bacteria, yeast, and animal cells, a single concatamer insert can be visualized with high resolution and fidelity $(75,84,93)$. In yeast and bacteria, the application of this technique provided new insights into the behavior of chromosomes during cell division. For example, sister chromatid separation in yeast was shown to occur in the absence of microtubules (84). This method has also been extended to track the movement of specific chromosomal locations within living cells (63). Using single-particle tracking with 3-D fluorescence microscopy, the movement of the LEU2 locus near the centromere of yeast chromosome III was followed by a GFP-LacI beacon. Consistent with previous work using fluorescence photobleaching recovery (1), the results from this work show that diffusion of chromosomes is slow and conforms to random Brownian movement without requiring active metabolism. This slow movement is constrained within an area of about $1 \%$ of the total nuclear volume. Together with the evidence from FISH studies that various loci of the genome can be found consistently in specific regions of the nucleus $(42,62,81,94)$, these new results from real-time measurements within living cells argue for a structured organization of chromosomes. Treatments that 
affect the integrity of the cytoskeleton result in an increased area of movement for the labeled chromosome, suggesting that the physical constraint within the nucleus is regulated at least in part by cytoplasmic components (63). Recently, Heun et al. (39) reported large, rapid ( $>0.5 \mu \mathrm{m}$ in $10 \mathrm{sec}$.) movements of tagged sites in budding yeast nuclei at the G1- and S-phases. These movements require ATP and are not seen in cells at the stationary phase, thus lending support to the hypothesis that they are correlated with transcription and/or chromatin remodeling activities in the cell.

A key advantage of the operator array tagging strategy is that specific sites in the genome can be visually tagged in live cells, which enables tracking of the labeled sequence in near real time as well as delineating its relative location to other sequences or subnuclear landmarks. However, there are two major drawbacks for this approach: (a) The size of the operator array [approximately 10 kilobase pairs (kbs) in the original lac $O$ array construct $(75,84)$ ] may perturb the local chromatin structure. Using smaller operator arrays with brighter auto fluorescent proteins may help to minimize this concern. (b) Alterations of global chromatin organization may occur due to interactions between multiple operator arrays. Because the operator arrays contain highly repetitive sequences, it is conceivable that multiple insertions of these arrays in the genome may cause significant changes in the organization of the genome. Aragon-Alcaide \& Strunnikov (4) reported a phenomenon in budding yeast for multiple inserted arrays of tet $O$ or lac $O$; they coined this "trans-association." These somatic associations are sequence specific and are independent of chromosomal location or sequence context. Fuchs et al. (28) subsequently showed evidence that the association between tet $O$ arrays may depend on expression of the TetR fusion protein.

The operator array strategy can be further adapted to examine other chromatinbased processes. For example, Tumbar \& Belmont (88) showed that by tethering a strong transcriptional activator domain (i.e., VP16 activating domain from the Herpes Simplex Virus transactivator) to the LacI DBD, large-scale movement of the $l a c O$ array from the nuclear periphery toward the interior can be visualized upon transactivator recruitment. Also, by linking a transcription cassette expressing a distinct color variant of GFP to an operator array tag, one can simultaneously image the location of the gene and the production of its protein production in real time (87).

We recently demonstrated the feasibilty of the operator array approach for chromatin tagging in transgenic plants $(49,51)$. Using a steroid-inducible gene expression system, we demonstrated that expression of a GFP-LacI-NLS fusion protein in Arabidopsis cells can result in the formation of discrete fluorescent spots in the presence of $l a c O$ concatamers but not in its absence (Figure 2) (49). The specificity of this apparent association was confirmed by inhibition of spot formation by IPTG addition, which disrupts LacI/lacO interaction. The association of the observed GFP fluorescent spots to the inserted lacO concatamers was also recently confirmed by FISH analyses (N. Kato, E. Lam, A. Pecinka \& I. Schubert, unpublished data). Thus, chromatin tracking can be used with confidence for 
sequence-specific tagging in plants. Using an Arabidopsis transgenic line that contains two linked insertions with $\mathrm{lac} O$ concatamers, the diffusion rate of chromatin in leaf guard cells was compared with that of pavement cells from the hypocotyl (51). It was found that in live Arabidopsis plants, chromatin has constrained diffusive motion in interphase cells with rates that range from about $1.25 \times 10^{-4} \mu \mathrm{m}^{2} \mathrm{~s}^{-1}$ in guard cells to $5.35 \times 10^{-5} \mu \mathrm{m}^{2} \mathrm{~s}^{-1}$ in pavement cell nuclei. These are similar values to those measured recently in Drosophila spermatocytes (89). We found that chromatin in pavement cell nuclei has on average more than six times the range of diffusion area compared to that of chromatin in guard cells. Although guard cell nuclei are about four to five times smaller than nuclei of pavement cells, the ratio of DNA content to nuclear volume is relatively constant, and the large increase in diffusible area in pavement cells cannot be attributed to a simple increase in the size of the nucleus. Further studies are needed to elucidate the precise cause for the different behavior of chromatin in these cell types.

One limitation that we have encountered in deploying this technique in plants is uneven and variable induction of the auto fluorescent fusion protein when using a chemical inducer. The reason for this is currently unclear. In studies with live plants, another limitation is that the thickness of the tissue of interest may preclude certain cells from high-resolution studies due to technical limitations at the microscopy level. At this point, Arabidopsis is ideal for the operator array approach in chromatin tracking because whole, live seedlings can be mounted and examined under the microscope. In addition, the well-characterized genome makes determining the integration site of the particular operator array relatively straightforward. However, it is possible to envision other model plant systems for deploying this technology. A clear advantage of intact plants is their immobile nature due to the presence of the cell wall. This characteristic presents an unique opportunity to study cells with defined lineage and a clear and reproducible axis of orientation relative to the body plan of the organism. The continued optimization of the controlled expression of brighter spectral variants of GFP as well as quantitative imaging techniques with plant tissues would likely enable future application of this technology to study different plant cell types under various developmental conditions.

\section{CONCLUDING REMARKS}

The present model of chromatin organization within the nucleus is of a dynamic state, with "territories" for each chromosome defined by their relative location to each other $(26,54,94,95)$. In the cell division cycle, chromosomes are duplicated during the S-phase and pulled to the two daughter cells during M-phase. Chromosome territories are re-established after cell division and once the cell is arrested in a long interphase, chromosome movement is restricted again $(1,40,61,63)$. Some nucleus-to-nucleus variations in the exact position for each locus relative to the rest of the genome can be expected. Because some random diffusion within each 
territory is possible $(1,63)$, there are time-dependent small positional differences in the relative coordinates of each locus within a single nucleus. These nucleusto-nucleus variations and the mobility of the established territories in the nuclei of interphase cells are data that will become available in the near future for different plant species as well as different cell types. By carrying out a genome-wide survey of these parameters, a comprehensive picture of the subnuclear architecture and its dynamics may be generated. Deploying multicolored DNA tagging systems by using spectrally distinct auto fluorescent proteins (AFPs) should allow us to test the feasibility of this approach. As a prelude to this effort in plant systems, we recently demonstrated that it is possible to simultaneously resolve four spectrally distinct AFPs in plant tissues (50). We also showed that it is possible to simultaneously image four distinct compartments in the same cell of a transgenic plant (Figure 3). Thus, we hope that this approach will be feasible in higher-resolution studies at the subnuclear level and will enable visualization of multiple defined loci in the genome and in live plants. Together with high-resolution FISH techniques using fully mapped and sequenced BAC clones of Arabidopsis and rice, these technologies promise to open an exciting new era in chromatin studies of higher plants that should provide us with new information on the principles underlying genome organization.

\section{ACKNOWLEDGMENTS}

We gratefully acknowledge the National Science Foundation's generous support (Project \# 077617) for our research on chromatin organization at Rutgers University. In addition, we thank A. Pecinka and I. Schubert for contributing unpublished data, and A. Straight, N.H. Chua, L. Meisel, J. Haseloff, and W. Gruissem for the gift of plasmids and vectors throughout our work. We also gratefully acknowledge constructive discussions with J. Sedat and W. Marshall during the course of our project.

\section{The Annual Review of Plant Biology is online at http://plant.annualreviews.org}

\section{LITERATURE CITED}

1. Abney JR, Cutler B, Fillbach ML, Axelrod D, Scalettar BA. 1997. Chromatin dynamics in interphase nuclei and its implications for nuclear structure. J. Cell. Biol. 7:145968

2. Abranches R, Beven AF, Aragon-Alcaide L, Shaw PJ. 1998. Transcription sites are not correlated with chromosome territories in wheat nuclei. J. Cell. Biol. 143:5-12

3. Ach RA, Gruissem W. 1994. A small nuclear GTP-binding protein from tomato suppresses a Schizosaccharomyces pombe cell-cycle mutant. Proc. Natl. Acad. Sci. USA 91:5863-67

4. Aragon-Alcaide L, Strunnikov AV. 2000. Functional dissection of in vivo interchromosome association in Saccharomyces cerevisiae. Nat. Cell Biol. 2:812-18

5. Askajaer P, Galy V, Hannak E, Mattaj IW. 2002. Ran GTPase cycle and importins alpha and beta are essential for spindle formation and nuclear envelope assembly in 
living Caenorhabditis elegans embryos. Mol. Biol. Cell 13:4355-70

6. Aufsatz W, Mette FM, van der Winden J, Matzke M, Matzke JM. 2002. HDA6, a putative histone deacetylase needed to enhance DNA methylation induced by double-stranded RNA. EMBO J. 21:683241

7. Bachman KE, Rountree MR, Baylin SB. 2001. Dnmt3a and Dnmt3b are transcriptional repressors that exhibit unique localization properties to heterochromatin. $J$. Biol. Chem. 276:32282-87

8. Bamba C, Bobinnec Y, Fukuda M, Nishida E. 2002. The GTPase Ran regulates chromosome positioning and nuclear envelope assembly in vivo. Curr. Biol. 12:503-7

9. Bannister AJ, Zegerman P, Partridge JF, Miska EA, Thomas JO, et al. 2001. Selective recognition of methylated lysine 9 on histone $\mathrm{H} 3$ by the HP1 chromo domain. $\mathrm{Na}$ ture 410:120-24

10. Barcellona ML, Cardiel G, Gratton E. 1990. Time-resolved fluorescence of DAPI in solution and bound to polydeoxynucleotides. Biochem. Biophys. Res. Commun. 170:270-80

11. Bass HW, Marshall WF, Sedat JW, Agard DA, Cande WZ. 1997. Telomeres cluster de novo before the initiation of synapsis: a three-dimensional spatial analysis of telomere positions before and during meiotic prophase. J. Cell. Biol. 137:518

12. Bennett MD. 1984. Gene Manipulation in Plant Improvement. In Stadler Genetics Symposium, 6th, ed. JP Gustafson. pp. 469502. Plenum Press

13. Boisnard-Lorig C, Colon-Carmona A, Bauch M, Hodge S, Doerner P, et al. 2001. Dynamic analyses of the expression of the HISTONE::YFP fusion protein in Arabidopsis show that syncytial endosperm is divided in mitotic domains. Plant Cell 13:495-509

14. Bornfleth H, Edelmann P, Zink D, Cremer T, Cremer C. 1999. Quantitative motion analysis of subchromosomal foci in living cells using four-dimensional microscopy. Biophys. J. 77:2871-86

15. Carlton PM, Cowan CR, Cande WZ. 2003. Directed motion of telomeres in the formation of the meiotic bouquet revealed by time course and simulation analysis. Mol. Biol. Cell 14:2832-43

16. Cavalli G, Paro R. 1998. Chromo-domain proteins: linking chromatin structure to epigenetic regulation. Curr. Opin. Cell Biol. 10:354-60

17. Chikashige Y, Ding DQ, Funabiki H, Haraguchi T, Mashiko S, et al. 1994. Telomere-led premeiotic chromosome movement in fission yeast. Science 264: 270-73

18. Copenhaver GP, Pikaard CS. 1996. RFLP and physical mapping with an rDNAspecific endonuclease reveals that nucleolus organizer regions of Arabidopsis thaliana adjoin the telomeres on chromosomes 2 and 4. Plant J. 9:259-72

19. Copenhaver GP, Pikaard CS. 1996. Two-dimensional RFLP analysis reveal megabase-sized clusters or rRNA gene variants in Arabidopsis thaliana, suggesting local spreading of variants as the mode for gene homogenization during concerted evolution. Plant J. 9:273-82

20. Cremer T, Kreth G, Koester H, Fink RH, Heintzmann R, et al. 2000. Chromosome territories, interchromatin domain compartment, and nuclar matrix: an integrated view of the functional nuclear architecture. Crit. Rev. Eukaryot. Gene Expr. 10:179212

21. Cremer T, Cremer C. 2001. Chromosome territories, nuclear architecture and gene regulation in mammalian cells. Nat. Rev. Genet. 2:292-301

22. Dawe RK. 1998. Meiotic chromosome organization and segregation in plants. Annu. Rev. Plant Physiol. Plant Mol. Biol. 49:371-95

23. Desel C, Jansen R, Dedong G, Schmidt T. 2002. Painting of parental chromatids in Beta hybrids by multi-color fluorescent in situ hybridization. Ann. Bot. 89:171-81 
24. Ekwall K, Nimmo ER, Javerzat JP, Borgstrom B, Egel R, et al. 1996. Mutations in the fission yeast silencing factors $\mathrm{clr}^{+}$and $\mathrm{rik}^{+}$disrupt the localization of the chromo domain protein Swi6p and impair centromere function. J. Cell Sci. 109:263748

25. Felsenfeld G, Groudine M. 2003. Controlling the double helix. Nature 421:448-53

26. Ferreira J, Paolella G, Ramos C, Lamond AI. 1997. Spatial organization of largescale chromatin domains in the nucleus: a magnified view of single chromosome territories. J. Cell. Biol. 139:1597-610

27. Fransz P, de Jong JH, Lysak M, Castiglione MR, Schubert I. 2002. Interphase chromosomes in Arabidopsis are organized as well defined chromocenters from which euchromatin loops emanate. Proc. Natl. Acad. Sci. USA 99:14584-89

28. Fuchs J, Lorenz A, Loidl J. 2001. Chromosome associations in budding yeast caused by integrated tandemly repeated transgenes. J. Cell Sci. 115:1213-20

29. Fussell CP. 1987. The Rabl orientation: a prelude to synapsis. In Meiosis, ed. PB Moens. pp. 275-99. Orlando, Fla.: Academic

30. Gendrel A-V, Lippman Z, Yordan C, Colot V, Martienssen RA. 2002. Dependence of heterochromatic histone H3 methylation patterns on the Arabidopsis gene DDM1. Science 297:1871-73

31. Gerlich D, Beaudouin J, Kalbfuss B, Daigle N, Eils R, Ellenberg J. 2003. Global chromosome positions are transmitted through mitosis in mammalian cells. Cell 112:75164

32. Gonzalez-Melendi P, Wells B, Beven AF, Shaw PJ. 2001. Single ribosomal transcription units are linear, compacted Christmas trees in plant nucleoli. Plant J. 27:223-33

33. Hall IM, Noma K, Grewal SI. 2002. RNA interference machinery regulates chromosome dynamics during mitosis and meiosis in fission yeast. Proc. Natl. Acad. Sci. USA 100:193-98

34. Heslop-Harrison JS, Bennett MD. 1990.
Nuclear architecture in plants. Trends Genet. 6:401-5

35. Heslop-Harrison JS, Murat M, Ogura Y, Schwarzacher T, Motoyoshi F. 1999. Polymorphisms and genomic organization of repetitive DNA from centromeric regions of Arabidopsis chromosomes. Plant Cell 11:31-42

36. Heslop-Harrison JS. 2000. Comparative genome organization in plants: from sequence and markers to chromatin and chromosomes. Plant Cell 12:617-35

37. Heslop-Harrison JS. 2003. Planning for remodelling: nuclear architecture, chromatin and chromosomes. Trends Plant Sci. 8:195-97

38. Hetzer M, Bilbao-Cortes D, Walther TC, Gruss OJ, Mattaj IW. 2000. GTP hydrolysis by Ran is required for nuclear envelope assembly. Mol. Cell 5:1013-24

39. Heun P, Laroche T, Shimada K, Furrer P, Gasser SM. 2001. Chromosome dynamics in the yeast interphase nucleus. Science 294:2181-86

40. Hilliker AJ, Appels R. 1989. The arrangement of interphase chromosomes: structural and functional aspects. Exp. Cell Res. 185:297-318

41. Hiraoka Y, Dernburg AF, Parmelee SJ, Rykowski MC, Agard DA, Sedat JW. 1993. The onset of homologous chromosome pairing during Drosophila melanogaster embryogenesis. J. Cell. Biol. 120:591-600

42. Hochstrasser M, Mathog D, Gruenbaum Y, Saurnweber H, Sedat JW. 1986. Spatial organization of chromosomes in the salivary gland nuclei of Drosophila melanogaster. J. Cell. Biol. 102:112-23

43. Holmquist GP, Kapitonov VV, Jurka J. 1998. Mobile genetic elements, chiasma, and the unique organization of betaheterochromain. Cytogenet. Cell Genet. 80:113-16

44. Houen A, Field BL, Saunders VA. 2001. Microdissection and chromosome painting of plant B chromosomes. Methods Cell Sci. 23:115-24

45. Jackson JP, Lindroth AM, Cao X, Jacobsen 
SE. 2002. Control of CpNpG DNA methylation by the KRYPTONITE histone $\mathrm{H} 3$ methyltransferase. Nature 416:556-60

46. Jeddeloh JA, Stokes, TL, Richards EJ. 1999. Maintenance of genomic methylation requires a SWI2/SNF2 protein. Nat. Genet. 22:94-97

47. Johnson LM, Cao X, Jacobsen SE. 2002. Interplay between two epigenetic marks: DNA methylation and histone $\mathrm{H} 3$ lysine 9 methylation. Curr. Biol. 12:1360-67

48. Kanda T, Sullivan KF, Wahl GM. 1998. Histone-GFP fusion protein enables sensitive analysis of chromosome dynamics in living mammalian cells. Curr. Biol. 8:37785

49. Kato N, Lam E. 2001. Detection of chromosomes tagged with green fluorescent protein in live Arabidopsis thaliana plants. Genome Biol. 2:(45)1-10

50. Kato N, Pontier D, Lam E. 2002. Spectral profiling for the simultaneous observation of four distinct fluorescent proteins and detection of protein-protein interaction via fluorescence resonance energy transfer in tobacco leaf nuclei. Plant Physiol. 129:931-42

51. Kato N, Lam E. 2003. Chromatin of endoreduplicated pavement cells has greater range of movement than that of diploid guard cells in Arabidopsis thaliana. J. Cell Sci. 116:2195-201

52. Kornberg RD, Lorch Y. 1999. Twenty-five years of the nucleosome, fundamental particle of the eukaryote chromosome. Cell 98:285-94

53. Kubista M, Akerman B, Norden B. 1987. Characterization of interaction between DNA and 4',6-diamidino-2-phenylindole by optical spectroscopy. Biochemistry 26:4545-53

54. Lamond AI, Earnshaw WC. 1998. Structure and function in the nucleus. Science 280:547-53

55. Lachner M, Jenuwein T. 2002. The many faces of histone lysine methylation. Curr. Opin. Cell Biol. 14:286-98

56. Lawrence JB, Singer RH, Marselle LM.
1989. Highly localized tracks of specific transcripts within interphase nuclei visualized by in situ hybridization. Cell 57:493502

57. Lindroth AM, Cao X, Jackson JP, Zilberman D, McCallum CM, et al. 2001. Requirement of CHROMOMETHYLASE3 for maintenance of $\mathrm{CpXpG}$ methylation. Science 292:2077-80

58. Lusser A. 2002. Acetylated, methylated, remodeled: chromatin states for gene regulation. Curr. Opin. Plant Biol. 5:437-43

59. Lysak MA, Fransz PF, Ali HB, Schubert I. 2001. Chromosome painting in Arabidopsis thaliana. Plant J. 28:689-97

60. Lysak MA, Pecinka A, Schubert I. 2003. Recent progress in chromosome painting of Arabidopsis and related species. Chromosome Res. 11:195-204

61. Manders EMM, Kimura H, Cook PR. 1999. Direct imaging of DNA in living cells reveals the dynamics of chromosome formation. J. Cell. Biol. 144:813-21

62. Marshall WF, Dernburg AF, Harmon B, Agard DA, Sedat JW. 1996. Specific interactions of chromatin with the nuclear envelope: positional determination within the nucleus in Drosophila melanogaster. Mol. Biol. Cell 7:825-42

63. Marshall WF, Straight A, Marko JF, Swedlow J, Dernburg A, et al. 1997. Interphase chromosomes undergo constrained diffusional motion in living cells. Curr. Biol. 7:930-39

64. Marshall WF, Fung JC, Sedat JW. 1997. Deconstructing the nucleus: global architecture from local interactions. Curr. Opin. Genet. Dev. 7:259-63

65. Marshall WF. 2002. Order and disorder in the nucleus. Curr. Biol. 12:R185-92

66. Martinez-Perez E, Shaw P, Moore G. 2001. The $P h l$ locus is needed to ensure specific somatic and meiotic centromere association. Nature 411:204-7

67. Mascetti G, Carrara S, Vergani L. 2001. Relationship between chromatin compactness and dye uptake for in situ chromatin stained with DAPI. Cytometry 44:113-19 
68. Merkle T, Haizel T, Matsumoto T, Harter K, Dallmann G, Nagy F. 1994. Phenotype of the fission yeast cell cycle regulatory mutant pim1-46 is suppressed by a tobacco cDNA encoding a small, Ran-like GTPbinding protein. Plant J. 6:555-65

69. Misteli T, Caceres JF, Spector DL. 1997. The dynamics of a pre-mRNA splicing factor in living cells. Nature 387:523-27

70. Nagele R, Freeman T, McMorrow L, Lee H. 1995. Precise spatial positioning of chromosomes during prometaphase: evidence for chromosomal order. Science 270:183135

71. Papa CM, Springer NM, Muszynski MG, Meeley R, Kaeppler SM. 2001. Maize chromomethylase Zea methyltransferase 2 is required for $\mathrm{CpNpG}$ methylation. Plant Cell 13:1919-28

72. Pelissier T, Tutois S, Tourmente S, Deragon JM, Picard G. 1996. DNA regions flanking the major Arabidopsis thaliana satellite are principally enriched in Athila retroelement sequences. Genetica $97: 141-51$

73. Pinkel D, Straume T, Gray JW. 1986. Cytogenetic analysis using quantitative, high-sensitivity, fluorescence hybridization. Proc. Natl. Acad. Sci. USA 83:293438

74. Probst AV, Fransz PF, Paszkowski J, Scheid OM. 2003. Two means of transcriptional reactivation within heterochromatin. Plant J. 33:743-49

75. Robinett CC, Straight A, Li G, Willheirn C, Sudlow G, et al. 1996. In vivo localization of DNA sequences and visualization of large-scale chromatin organization using Lac operator/repressor recognition. $J$. Cell. Biol. 135:1685-700

76. Round EK, Flowers SK, Richards EJ. 1997. Arabidopsis thaliana centromore regions: genetic map positions and repetitive DNA structure. Genome Res. 7:1045-53

77. Saalbach G, Christov V. 1994. Sequence of a plant cDNA from Vicia faba encoding a novel Ran-related GTP-binding protein. Plant Mol. Biol. 24:969-72

78. Schotta G, Ebert A, Krauss V, Fischer A,
Hoffmann J, et al. 2002. Central role of Drosophila SU(VAR)3-9 in histone H3-K9 methylation and heterochromatic gene silencing. EMBO J. 21:1121-31

79. Schrock E, du Manoir S, Veldman T, Schoell B, Wienberg J, et al. 1996. Multicolor spectral karyotyping of human chromosomes. Science 273:494-97

80. Schwarzacher T, Heslop-Harrison JS. 1994. Direct fluorochrome-labeled DNA probes for direct fluorescent in situ hybridization to chromosomes. Methods Mol. Biol. 28:167-76

81. Shelby RD, Hahn KM, Sullivan KF. 1996. Dynamic elastic behavior of $\alpha$-satellite DNA domains visualized in situ in living human cells. J. Cell. Biol. 135:545-57

82. Soppe WJJ, Jasencakova Z, Houben A, Kakutani T, Meister A, et al. 2002. DNA methylation controls histone H3 lysine 9 methylation and heterochromatin assembly in Arabidopsis. EMBO J. 21:654959

83. Speicher MR, Ballard GS, Ward DC. 1996. Karyotyping human chromosomes by combinatorial multi-fluor FISH. Nat. Genet. 12:368-75

84. Straight AF, Belmont AS, Robinett CC, Murray AW. 1996. GFP tagging of budding yeast chromosomes reveals that proteinprotein interactions can mediate sister chromatid cohesion. Curr. Biol. 6:1599-608

85. Tariq M, Saze H, Probst AV, Lichota J, Habu Y, Paszkowski J. 2003. Erasure of $\mathrm{CpG}$ methylation in Arabidopsis alters patterns of histone H3 methylation in heterochromatin. Proc. Natl. Acad. Sci. USA 100:8823-27

86. Trask BJ. 1991. Fluorescence in situ hybridization: applications in cytogenetics and gene mapping. Trends Genet. 7:14954

87. Tsukamoto T, Hashiguchi N, Janicki SM, Tumbar T, Belmont AS, Spector DL. 2000. Visualization of gene activity in living cells. Nat. Cell Biol. 2:871-78

88. Tumbar T, Belmont AS. 2001. Interphase movements of a DNA chromosome region 
modulated by VP16 transcriptional activator. Nat. Cell Biol. 3:134-39

89. Vazquez J, Belmont AS, Sedat JW. 2002. The dynamics of homologous chromosome pairing during male Drosophila meiosis. Curr. Biol. 12:1473-83

90. Volpe TA, Kidner C, Hall IM, Teng G, Grewal SI, Martienssen RA. 2002. Regulation of heterochromatic silencing and histone H3 lysine- 9 methylation by RNAi. Science 297:1833-37

91. Vongs A, Kakutani T, Martienssen RA, Richards EJ. 1993. Arabidopsis thaliana DNA methylation mutants. Science 260:1926-28

92. Walter J, Schermelleh L, Cremer M, Tashiro S, Cremer T. 2003. Chromosome order in HeLa cells changes during mitosis and early G1, but is stably maintained during subsequent interphase stages. J. Cell. Biol. 160:685-97

93. Webb CD, Teleman A, Gordon S, Straight A, Belmont A, et al. 1997. Bipolar localization of the replication origin regions of chromosomes in vegetative and sporulating cells of B. subtilis. Cell 88:667-74

94. Zalensky AO, Allen MJ, Kobayashi A, Zalenskaya IA, Balhorn R, Bradbury EM. 1995. Well-defined genome architecture in the human sperm nucleus. Chromosoma 103:577-90

95. Zink D, Cremer T, Saffrich R, Fischer R, Trendelenburg MF, et al. 1998. Structure and dynamics of human interphase chromosome territories in vivo. Hum. Genet. 102:241-51 


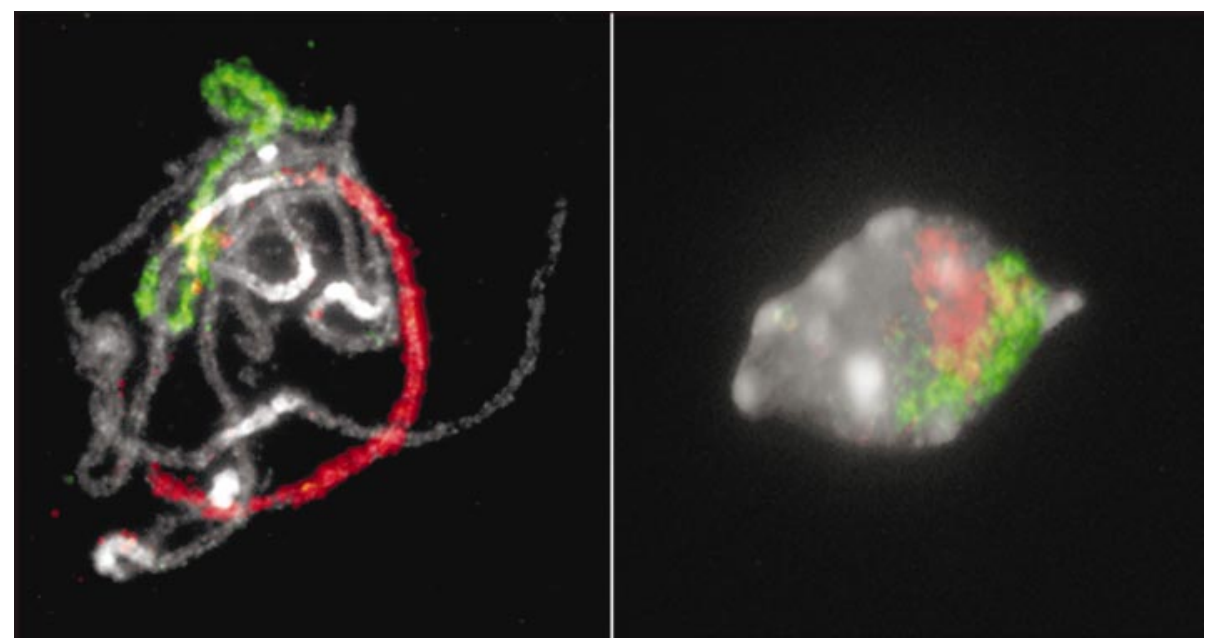

Figure 1 Painted Arabidopsis thaliana Chromosome 1 in the pachytene stage of meiotic cell division (left panel) and Chromosome 1 territories in interphase nuclei isolated from root tissues (right panel). One hundred and eighty-three Chromosome 1-specific BAC clones were used to paint the chromosome. Chromatids and nuclei are stained by DAPI (white). The top arm is labeled by Texas Red dye (red) and the bottom arm of the chromosome is labeled by Alexa 488 dye (green). The images were kindly provided by A. Pecinka in the Schubert laboratory at the IPK in Gatersleben. 


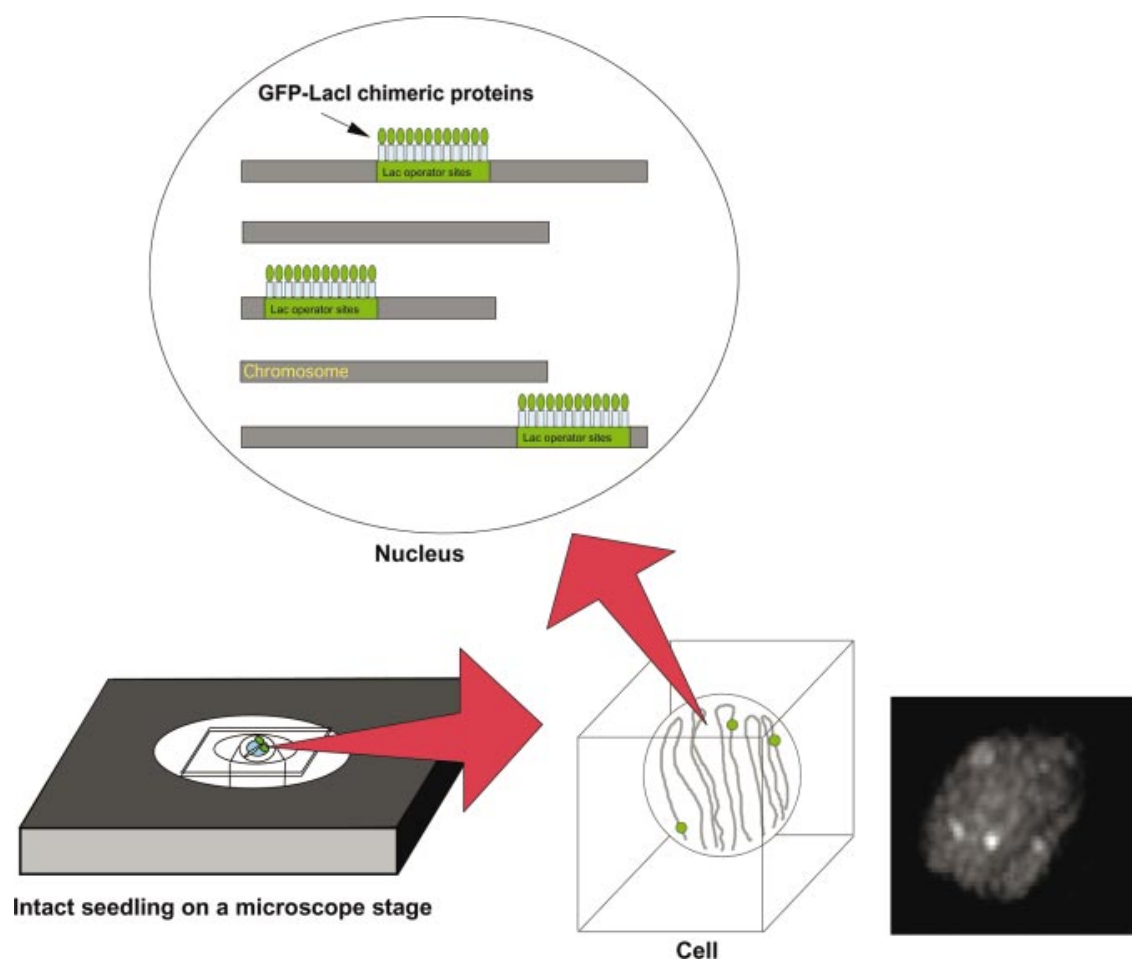

Figure 2 Application of the Green Fluorescent Protein (GFP) as an in vivo tag of specific chromosomal regions in living plants. Concatamers of the lac operator are inserted in different chromosomal loci (Lac operator sites). GFP-LacI fusion proteins are then expressed in the nucleus using an inducible promoter system. The fusion proteins recognize the operator sequence, and the bound arrays are visualized as bright fluorescent spots in the nuclei with a fluorescence microscope. An image of a transgenicArabidopsis nucleus is shown on the bottom panel to illustrate the data that can be obtained. Three bright fluorescent spots, which correspond to lac operator site arrays inserted in the chromosomes, are detected over background fluorescence that is caused by presumably unbound GFP-LacI fusion proteins in the nucleus. 


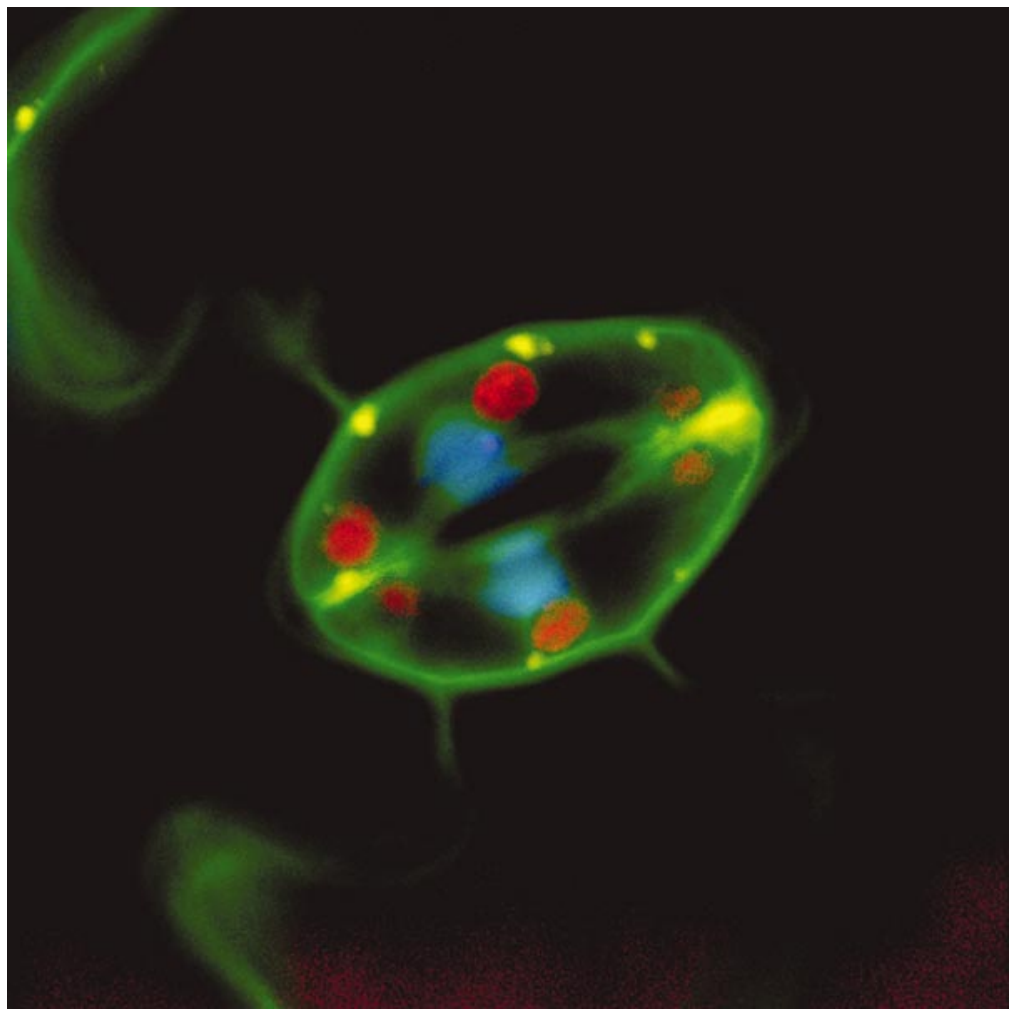

Figure 3 Detection of four distinct compartments in a single cell. An image of stomatal guard cells of a transgenic Arabidopsis is taken using a fluorescence microscope. Nuclei are tagged with GAL4-CFP (cyan fluorescent protein), shown in blue; mitochondria is tagged with CoxIV -YFP (cytochrome c oxidase subunit IV-yellow fluorescence protein), shown in yellow; and plasma membrane is tagged with calmodulin CaM53BD-GFP, shown in green. Chloroplasts are detected via their auto fluorescent chlorophylls, shown in red. The plastids are tagged with DsRed2 protein fused to plastid-targeted RecA with similar results (N. Kato \& E. Lam, unpublished results). 
R

Volume 55, 2004

\section{CONTENTS}

AN UnForeseen Voyage to THE World of PHYTOCHROMES, Masaki Furuya

Alternative NAD(P)H DehydROGENASES OF Plant

MitochONDRIA, Allan G. Rasmusson, Kathleen L. Soole, and Thomas E. Elthon

DNA MeThylation AND EPIGENETiCs, Judith Bender

PhosphoenolPyRUVATE CARBOXYlase: A NEW ERA OF STRUCTURAl BIOlOGY, Katsura Izui, Hiroyoshi Matsumura, Tsuyoshi Furumoto, and Yasushi Kai

Metabolic Channeling in Plants, Brenda S.J. Winkel

RHAMNOGALACTURONAN II: STRUCTURE AND FUNCTION OF A Borate Cross-LinKed Cell Wall PeCtic PolysaCCHARIDE, Malcolm A. O'Neill, Tadashi Ishii, Peter Albersheim, and Alan G. Darvill

NATURALLY OCCURRING GENETIC VARIATION IN ARABIDOPSIS

THALIANA, Maarten Koornneef, Carlos Alonso-Blanco, and Dick Vreugdenhil

Single-Cell $\mathrm{C}_{4}$ Photosynthesis Versus the Dual-Cell (KRANZ) PARADIGM, Gerald E. Edwards, Vincent R. Franceschi, and Elena $V$. Voznesenskaya

MoleCUlar Mechanism of GibBerellin Signaling IN Plants, Tai-ping Sun and Frank Gubler

Phytoestrogens, Richard A. Dixon

Decoding $\mathrm{Ca}^{2+}$ Signals Through Plant Protein KinAsEs, Jeffrey F. Harper, Ghislain Breton, and Alice Harmon

Plastid Transformation in Higher Plants, Pal Maliga

Symbioses of GRASSES WITH SEEDBORNE FunGAL ENDOPHYTES, Christopher L. Schardl, Adrian Leuchtmann, Martin J. Spiering

TRANSPORT MECHANISMS FOR ORGANIC FORMS OF CARBON AND NITROGEN BETWEEN SOURCE AND SINK, Sylvie Lalonde, 
Reactive OXYGen SPecies: Metabolism, Oxidative StRess,

AND Signal TRANSDUCTION, Klaus Apel and Heribert Hirt

The Generation of $\mathrm{Ca}^{2+}$ Signals in Plants,

Alistair M. Hetherington and Colin Brownlee

BIOSYNTHESIS AND ACCUMULATION OF STEROLS, Pierre Benveniste

How Do CRop Plants Tolerate ACID SOILS? MECHANISMS OF

ALUMINUM TOLERANCE AND PHOSPHOROUS EFFICIENCY,

Leon V. Kochian, Owen A. Hoekenga, and Miguel A. Piñeros

VIGS VeCTORS FOR GENE SLIENCING: MANY TARGETS, MANY TOOLS, Dominique Robertson

495

GENETIC REGULATION OF TIME TO FLOWER IN ARABIDOPSIS THALIANA, Yoshibumi Komeda

VisuALIZING CHROMOSOME STRUCTURE/ORGANIZATION, Eric Lam, Naohiro Kato, and Koichi Watanabe

The UbiQuitin 26S Proteasome Proteolytic PathWay, Jan Smalle and Richard D. Vierstra

Rising ATMOSPHERIC CARBON DiOXIDE: Plants FACE THE FUTURE, Stephen P. Long, Elizabeth A. Ainsworth, Alistair Rogers, and Donald R. Ort

INDEXES

Subject Index

Cumulative Index of Contributing Authors, Volumes 45-55

661

Cumulative Index of Chapter Titles, Volumes 45-55

666

\section{ERRATA}

An online log of corrections to Annual Review of Plant Biology chapters may be found at http://plant.annualreviews.org/ 